\title{
UMA TÉCNICA PARA MEDIR E ACOMPANHAR A MOTIVAÇÃO INTRÍNSECA DE TIMES ÁGEIS
}

\author{
Romildo F. Lima Júnior ${ }^{1,2}$, Samira S. Amaral Ribeiro ${ }^{1,3}$, Sandra R. Chaves \\ Carvalho ${ }^{1}$, Morgana V. Rodrigues ${ }^{1}$, Albert Schilling Gomes ${ }^{1}$ \\ ${ }^{1}$ Instituto Atlântico \\ Rua Chico Lemos, 946 - Cidade dos Funcionários - Fortaleza - Ceará - Brasil \\ ${ }^{2}$ Faculdade 7 de Setembro (FA7) \\ Av. Almirante Maximiniano da Fonseca, 1395 - Fortaleza - CE - Brasil \\ ${ }^{3}$ Universidade de Fortaleza (UNIFOR) \\ Av. Washington Soares, 1321 - Fortaleza - Ceará - Brasil \\ \{romildo_lima, samira_ribeiro, carvalho_sandra, morgana_rodrigues, \\ albert\}@ātlantico.com.br, romildoflj@gmāil.com, samirarb̄egmail.com
}

Resumo. Manter os membros da equipe motivados é um desafio constante. A identificação e o monitoramento das motivações intrínsecas tem um nível mais elevado de complexidade quando comparado às motivações extrínsecas. Essa dificuldade pode ser mais acentuada quando nos referimos a projetos ágeis, devido às constantes mudanças. Este artigo trata do uso da técnica: Measuring Motivator Cycle (MMC). MMC é uma adaptação da técnica Moving Motivators do Management 3.0, onde o diferencial é a atribuição de pontos para as motivações intrínsecas, resultando em um indicador que calcula a média geral de motivação da equipe, tornando o acompanhamento da motivação algo mais papável.

Abstract. Keep members motivated team is a constant challenge. Identification and monitoring of intrinsic motivations have a higher level of complexity when compared to extrinsic. This difficulty may be sharper when we refer to Agile projects due to constant changes. This article deals with the use of a technique: Measuring Motivator Cycle (MMC). MMC is an adaptation of the technical Moving Motivators from Management 3.0, where the differential is the allocation of points to the intrinsic motivations, resulting in an indicator that calculates the overall average team motivation, making the monitoring of motivation something more tangible.

\section{Introdução}

Assim como o ambiente empresarial, a gestão de projetos vem passando por diversas mudanças ao longo dos anos. Toda essa transformação vem demonstrando lacunas nos modelos de gestão e provocando a renovação desses modelos e paradigmas [Knapik 2011], a fim de que as empresas potencializem seus lucros. 
Diante desse cenário de transformações empresariais nacional e internacional, uma das principais descobertas vem sendo o indivíduo. Observa-se que o sucesso dos projetos não está relacionado apenas aos aspectos técnicos, mas também às características comportamentais dos indivíduos envolvidos nos projetos. Diversos são os ganhos em performance para os projetos e rendimentos financeiros para as organizações que valorizam mais e mais seus colaboradores [Ferreira e Ramos 2010] [Chiavenato 2008].

Para acompanhar a evolução das gerações de indivíduos e as diversas mudanças sociais as quais estamos expostos, faz-se necessário que a gestão de projetos abra mais espaço para a área de gestão de pessoas, ou recursos humanos, dedicando tanto tempo ou mais do que o dedicado às demais áreas do conhecimento da gestão de projetos tradicional [PMI 2011].

Diversas são as problemáticas que podem surgir devido ao não monitoramento das motivações de indivíduos envolvidos no projeto, tais como falta de comprometimento, baixa produtividade e até mesmo aumento da taxa de turnorver [Thatcher et al 2006].

Em uma empresa de pesquisa e desenvolvimento não é diferente. Observa-se tal cenário em projetos que seguem metodologias ágeis, no Instituto Atlântico. O Instituto Atlântico é uma instituição de pesquisa e desenvolvimento de soluções em tecnologia da informação, que busca a melhoria contínua da qualidade de seus processos de software, visando o aumento de produtividade, cumprimento de prazos estabelecidos, redução de custos do produto final e, sobretudo, atender cada vez melhor a seus clientes [Pires 2004].

O gerenciamento baseado em pessoas está intimamente ligado a um dos princípios, do manifesto ágil [Beck et al 2001]: Indivíduos e interação entre eles mais que processos e ferramentas. Quando o manifesto cita que é preciso ter maior atenção a indivíduos interpreta-se como uma preocupação com pessoas. A manutenção de profissionais satisfeitos com os fatores que compõe seu trabalho e o ambiente que o permeia faz parte desta preocupação. A satisfação com o trabalho leva ao contentamento e estar contente com algo compele pessoas ao engajamento, pois o indivíduo busca contentamento. Estar motivado é o ato de ser movido para algo, ou seja, ser compelido a este algo [Ryan e Deci 2000].

Sendo assim, observa-se a necessidade de técnicas que auxiliem as organizações a motivar seus times, mensurando e acompanhando sua motivação, bem como promovendo ações para melhoria desse relacionamento, o que é o foco deste trabalho. Desse modo, é possível enxergar os obstáculos que barram o crescimento dos indivíduos e, em suas devidas proporções, dos projetos e das empresas.

Este artigo trata de um relato de experiência aplicado no Instituto Atlântico. Com base neste contexto, foi desenvolvida e aplicada a técnica Measuring Motivation Cycle (MMC). O objetivo desta técnica é deixar mais tangível a motivação de membros do time, quantificando-a, o que permitirá medi-la e monitorá-la individualmente ou em grupo. 
$\mathrm{O}$ artigo está dividido em 7 seções, incluindo esta Introdução. A Seção 2 apresenta uma reflexão sobre motivações e seus tipos. A Seção 3 apresenta algumas abordagens para monitoramento de motivação de indivíduos. A Seção 4 apresenta a técnica MMC proposta neste artigo. Na seção 5 é descrito o cenário no qual a nova técnica foi aplicada como estudo de caso e na Seção 6 descrevemos os resultados deste estudo. A Seção 7 discute as principais contribuições deste artigo e suas conclusões, além de propor trabalhos futuros.

\section{Motivações do Indivíduo}

Algumas teorias relacionadas à motivação têm guiado por vários anos o trabalho de gestores. Temos como duas das mais conhecidas: Hierarquia de necessidades de Maslow e teoria dos fatores higiênicos de Herzberg [Ramlall 2004].

Maslow acredita que existem pelo menos cinco objetivos os quais podem ser referidos como as necessidades básicas: fisiológica, segurança, amor, estima e auto realização [Ramlall 2004]. Segundo Maslow, o ser humano estaria em uma busca constante por estes objetivos e em forma hierárquica de importância. Desta forma o objetivo seguinte só poderia ser atingido se o anterior estiver satisfeito.

Já Herzberg, ao contrário de Maslow, mapeou não apenas fatores que levariam a motivação, mas também fatores que levariam a desmotivação [Periard 2011]. Através de questionários conduzidos com os membros de fábricas ao longo de anos, ele mapeou grupos de "motivadores" e "desmotivadores", estes últimos sendo chamados de fatores higiênicos. A ausência de fatores higiênicos levaria a desmotivação, mas o contrário não necessariamente levaria a motivação.

\subsection{Tipos de motivação do indivíduo}

Estar motivado é o ato de ser movido para algo [Ryan e Deci 2000]. Uma pessoa que não sente impulso ou inspiração para algo é dita desmotivada, enquanto que uma pessoa que está energizada ou está em movimento para o objetivo é dita motivada.

A motivação pode ser classificada em dois tipos ou orientações: extrínsecas ou intrínsecas. Estes tipos são definidos em relação aos indivíduos e suas atividades. Assim alguém sente-se intrinsecamente ou extrinsecamente motivado para realizar uma atividade [Ryan e Deci 2000].

Como motivações extrínsecas entendem-se as motivações externas tais como premiações, comandos ou ordens, punições entre outros. Tais motivações tem origens variadas como por exemplo salários, leis, regras de uma empresa entre outras.

Apesar de ter um papel importante, tais motivações tem um prazo de validade e podem gerar dependência, o que são considerados efeitos colaterais [Appelo 2011]. Quando dizemos "prazo de validade" nos referimos ao tempo ao final do qual tal motivação deixa de influenciar positivamente uma pessoa. Por exemplo, um salário maior sofre influência da inflação ou o profissional pode aumentar seu padrão de vida fazendo com que haja uma nova necessidade de progressão salarial dentro de algum tempo. Usando ainda o mesmo exemplo anterior para demonstrar a geração de 
dependência, supondo que a cada um ano um profissional venha recebendo progressões salariais e em determinado momento uma crise financeira impactou em congelamento dos salários. Isto poderá levar a uma "crise de abstinência" naquele profissional que não viu seu rendimento crescer como de costume. Em um nível mais crítico, temos o caso de uma empresa que atrasa os salários de seus profissionais. Sem tais rendimentos, a tendência é que a moral de todos baixe devido a dependência destes valores para manutenção das necessidades de suas famílias (fatores higiênicos de Herzberg).

Motivações intrínsecas são consideradas aquelas que surgem de uma pessoa de acordo com suas preferências ou necessidades. Quando uma pessoa sente-se atraída por uma determinada atividade independente de impulsos externos como ordens, premiações ou encorajamentos, é dito que tal pessoa está intrinsecamente motivada para aquela atividade.

Como exemplo de motivação intrínseca podemos citar o desejo de aprender e nos aprimorar nas mais variadas áreas de conhecimento, de acordo com os interesses de cada um. Alguns podem sentir-se motivados para artes, outros para ciências, matemática, entre outros. Percebe-se que os conceitos dos dois tipos de motivações são antagônicos.

As motivações intrínsecas são aquelas que fazem com que um profissional levante todos os dias de sua cama para vir ao trabalho [Appelo 2011]. Tais motivações são tão intimamente relacionadas com os valores e formações de uma pessoa que, não teriam um prazo de validade, ou seja, enquanto alguém se sente intrinsecamente motivado a realizar uma atividade a executará da melhor forma possível ao seu alcance. Reiss classificou em os tipos de motivações intrínsecas em dezesseis (Aceitação, Atividade Física ou Exercício, Curiosidade, Poder, Comer, Romance, Família, Economizar, Honra, Contato Social, Idealismo, Posição, Independência, Tranquilidade, Ordem e Vingança) [Reiss 2002].

\section{O que se Usa Para Monitorar as Motivações do Indivíduo}

Os Millennials ou geração Y é a geração dos nascidos entre 1980 e 2000 e é composta de pessoas conectadas 24 horas por dia e sete dias por semana, portanto o trabalho está constantemente presente, embora eles acreditem que o mesmo faz parte da sua vida, mas não representa a mesma, pois eles prezam o seu tempo livre [Lipkin e Perrymore 2010]. Eles buscam a ideia que vai mudar as suas vidas e montam Startups em tentativas de atingir o sucesso [BOX1824 2013]. Para liderar este tipo de profissional é preciso promover uma relação de troca com bons resultados para organização e profissionais [De Oliveira 2015].

Atualmente grande parte dos membros de times de desenvolvimento de software é composto por membros da geração $\mathrm{Y}$, portanto é necessário compreender estes profissionais para monitorar sua motivação. Seguem algumas técnicas, que podem realizar o monitoramento das motivações destes indivíduos:

I) Motivational Structure Questionaire (MSQ) apresenta uma abordagem guiada pelos valores do indivíduo pesquisado. O MSQ visa orientar a quem o está respondendo 
a identificar o que considera mais importante, suas principais preocupações, para que as respostas sejam guiadas por estas preocupações [Cox e Klinger 2004].

II) A técnica Personal Concerns Inventory (PCI), é uma versão simplificada do MSQ, a qual traz todas as respostas para uma escala de zero a dez permitindo colocar os resultados em médias. Desta forma o questionário pode ser aplicado via computador, por exemplo [Cox e Klinger 2004].

III) Moving Motivators é uma técnica do Management 3.0. Considerada a nova forma de gerenciar pelo pai do termo, o holandês Jurgen Appelo, o termo Management 3.0, é focado em pessoas e na forma como estas interagem para formar times de desenvolvimento de software. O Management 3.0 está estruturado em seis visões, dentre elas destaca-se a visão "Energizar pessoas", destinada para monitorar e aumentar a motivação destas pessoas [Appelo 2011].

Uma pessoa insatisfeita não consegue produzir de forma satisfatória [Jiang et al 2009], devido a isto, o foco maior da gestão deve estar nas pessoas [Appelo 2011].

A partir do uso de um subconjunto de motivações intrínsecas mapeadas por Pink [Pink 2011], Reiss [Reiss 2002] e Ryan e Deci [Ryan e Deci 2000], Appelo criou a ferramenta Moving Motivators [Appelo 2011]. Tal ferramenta foi proposta para usos em reuniões onde lideranças pudessem identificar as principais necessidades intrínsecas dos membros do time. Foi observado que dos dezesseis desejos intrínsecos de Reiss [Reiss 2002], um subconjunto de dez poderia ser influenciado no trabalho e fazer com que as pessoas sintam-se cada vez mais motivadas. As demais seis são muito pessoais para serem influenciadas, como Romance. A seguir citamos os dez Moving Motivators:

- Curiosidade: A necessidade de pensar, investigar e aprender;

- Honra: Ser leal a um grupo e ver seus valores pessoais serem refletidos nas pessoas do seu convívio. Ex: Pessoas que trabalham gratuitamente para instituições de caridade valorizam ajudar pessoas e sentem-se motivadas a trabalhar com outras pessoas que acreditam nisto;

- Aceitação: A necessidade de aprovação do seu modo de ser e agir;

- Maestria: A necessidade de sentir-se capaz ou produtivo. Por exemplo: Um profissional que encara de forma negativa a proposta para uma posição em área diferente da sua formação, pois sabe que haverá grande esforço pessoal de aprendizado e grande espaço de tempo para que o mesmo atinja o mesmo nível de produtividade que possuía na posição anterior é motivado por maestria;

- Poder: A necessidade de influenciar a vontade de outras pessoas. Ex: Pessoas que desejam participar de decisões sendo consultadas ou influenciando outras pessoas para que tomem atitudes do seu interesse, almejam bom nível de poder;

- Liberdade: A necessidade da individualidade. Possuir um determinado nível de autonomia em algum contexto;

- Relacionamento: A necessidade de ter amigos e relacionar-se bem com outros membros do seu convívio;

- Ordem: O desejo de sentir o ambiente estável e dentro dos padrões de organização desejáveis; 
- Propósito: A necessidade de estar sendo movido para realização de um propósito;

- Posição: A necessidade do destaque social ou reconhecimento pelos atos realizados;

Os desejos intrínsecos acima foram organizados em um baralho. Em um primeiro momento o profissional organiza os motivadores por ordem de importância da esquerda para direita.

Fazendo uma explicação do porquê de cada um dos cartões ficarem nesta ordem ambos compreendem o contexto atual do profissional. $\mathrm{O}$ aplicador do método pode então propor uma série de situações e mudanças, onde o profissional poderá mover para cima o cartão afetado, caso o mesmo se sinta mais motivado, ou ainda mover o cartão para baixo, caso contrário. Se os cartões posicionados como mais importantes forem afetados positivamente, recomenda-se promover tal mudança e do mesmo modo não mudar se os cartões mais importantes baixarem [Appelo 2011].

Dizer se alguém está motivado pode ser algo muito difícil. A identificação do nível de motivação intrínseca pode ser determinante para manutenção de um bom rendimento de um profissional e de um time. Lideranças de grupos de pessoas, estão constantemente buscando formas de motivar pessoas e acompanhar tal nível de forma a apresentar propostas de ações para obter times de alta performance. Performance em times pode ser determinada por uma função de:

Performance $=\mathrm{F}$ (habilidade $\mathrm{x}$ ambiente de trabalho $\mathrm{x}$ motivação) [Castka et al 2001, apud Stott e Walker 1995]

Vemos que duas das dimensões as quais compõe a função que determina a performance em times estão intimamente ligadas com os Moving Motivators [Appelo 2011]: ambiente de trabalho e motivação. Muitos membros de times buscam motivação através de um bom ambiente de trabalho, o que pode ser provido por motivações intrínsecas contempladas na ferramenta do Management 3.0. Um bom trabalho tem como critérios: cargas de trabalho quantitativas e qualitativas, oportunidades para controle no trabalho, claros papéis de trabalho balanceados por outros papéis e apoio a iteração social [Lindströn 1994]. Claramente é possível mapear alguns destes mesmos critérios nos motivadores ordem e relacionamento.

Tais pontos apenas reforçam a necessidade de monitorar as motivações dos times e este é o alvo da técnica apresentada neste trabalho. A falta de um indicador para acompanhar o status das motivações intrínsecas pode, por exemplo, resultar no aumento do turnover (taxa de rotatividade de pessoas que compõem um quadro de funcionários) de uma empresa [Thatcher et al 2006].

\section{Measuring Motivator Cycle (MMC)}

Esta adaptação consiste na atribuição de pesos a cada um dos principais motivadores destacados pelo profissional analisado. Assim seguimos todos os passos da metodologia 
proposta originalmente até o momento em que temos todas as cartas ordenadas por prioridade.

Selecionamos assim as cinco motivações mais importantes para o profissional e com isso passamos a solicitar ao mesmo que indique para cada carta o nível provido para cada motivação, dado o contexto em que se encontra na organização, e uma pontuação de um a três, onde um é o menor nível de motivação e três o maior. Por exemplo, supondo que o profissional informou ordem de prioridade apresentada na Tabela 1, de cima para baixo, a coluna pontuação indica qual nível de motivação no contexto atual para os cinco principais fatores motivacionais.

Tabela 1: Exemplo de coleta de níveis de motivação

\begin{tabular}{|l|l|}
\hline \multicolumn{1}{|c|}{ Motivador } & \multicolumn{1}{c|}{ Pontuação } \\
\hline Objetivo & 3 \\
\hline Maestria & 1 \\
\hline Relacionamento & 3 \\
\hline Ordem & 3 \\
\hline Liberdade & 3 \\
\hline Curiosidade & \\
\hline Posição & \\
\hline Aceitação & \\
\hline Honra & \\
\hline Poder & \\
\hline
\end{tabular}

Coletados os valores para cada carta, o facilitador vai passar a analisar em conjunto com o profissional quais os motivos para os níveis mais baixos de motivação e assim propor em conjunto ações para elevar estes níveis. O somatório da pontuação representa o nível de motivação do profissional. Seguindo o exemplo apontado na Tabela 1, o foco seria o motivador maestria, podendo talvez prover treinamento para o profissional, oportunidades de atuar em outras frentes dentro de sua zona de conforto, entre outras ações.

É definido um período para atuar no plano de ações e após este período executase novamente o processo de coleta para observação da efetividade do plano.

Reforçamos que para evitar que as respostas do profissional sejam tolhidas por qualquer tipo de receio, é necessário que sempre seja realizado um esclarecimento prévio quanto ao objetivo do uso da ferramenta, demonstrando que o foco é a preocupação com o profissional e que a finalidade é fazer com que suas motivações sejam mais bem tratadas e acompanhadas. 


\section{Estudo de Caso}

A técnica MMC foi aplicada em três times de projetos distintos, em três ciclos. Para os três times, foram realizadas três coletas no intervalo de 2 meses cada. Todas as coletas foram realizadas através de reuniões individuais entre o gerente de projeto e os membros do time, o que levava entre cinco e dez minutos cada reunião. As primeiras apresentaram um volume de tempo um pouco maior para que fosse possível explicar a dinâmica.

Apresentados a seguir os detalhes dos três times:

a) Time 1

O projeto do time 1 realizava desenvolvimento e testes de software e tinha por objetivo realizar adaptações em uma ferramenta legada de um parceiro. Os membros desse time frequentemente apresentavam queixas e solicitavam a saída do projeto. Os motivos que levavam a desmotivação do time foram:

- Projeto apresentava baixo nível de aprendizado;

- A ferramenta apresentava código mal estruturado;

- Cliente frequentemente demandava mais escopo de testes automatizados para os membros do time;

- Grande número de bugs a serem corrigidos.

Os cinco componentes do time, sendo três desenvolvedores e testadores e dois testadores, apresentavam pouca experiência, mas grande vontade de aprender. Todos os participantes foram membros da geração Y.

No começo do ano de 2016 houve uma mudança na composição: o membro mais experiente deixou o time, posto que suas habilidades foram requeridas em outro projeto e o mesmo já solicitava a mudança há algum tempo.

b) Time 2

O projeto ao qual o time em estudo pertencia tinha por objetivo realizar uma prova de conceito de uma solução de inovação tecnológica para um dos clientes mais importantes da empresa, através de investigações e desenvolvimento de software de forma iterativa e incremental de um conjunto de funcionalidades. Os motivos que levavam a desmotivação do time foram:

- Receio de não atender a expectativa do cliente pois o time foi reduzido à metade;

- Baixa confiança do time em relação a viabilidade da proposta;

- Etapa de investigações e validação das propostas muito longa;

- Cliente externo (americano): receio de não absorver as demandas devido as restrições idiomáticas e culturais.

A formação inicial desse time era composta por três desenvolvedores, sendo dois deles mais experientes nas tecnologias e no negócio e um deles menos experiente. Podese considerar um time misto que possuía membros da geração $\mathrm{X}$ e $\mathrm{Y}$. 
No mês de abril do ano de 2016 houve uma mudança na composição do time: entrou um novo membro, sendo uma pessoa com experiência dentro da empresa e com algum conhecimento nos negócios de atuação. Este profissional estava muito ansioso com a mudança, pois o mesmo estava apresentando dificuldade de adaptação no seu time anterior.

c) Time 3

$\mathrm{O}$ projeto em questão já estava em andamento a aproximadamente um ano e tratava de uma pesquisa em estágio intermediário. Todos os membros apresentavam grande experiência, sendo todos membros da geração Y. Já havia a sensação de bom nível de motivação geral, mas houve pontos a tratar. Algumas características do projeto:

- Projeto apresentava tecnologia extremamente atual;

- Havia necessidade de aprendizado de técnicas acadêmicas não muito presentes na vida profissional de todos os membros;

- Havia nível frequente de mudanças nos requisitos e prioridades das entregas;

- Provas de conceito eram grande maioria das atividades.

\section{Resultados}

Após aplicação da técnica para os três times, foi realizada a quantificação da motivação média e com isso foi feito o seu acompanhamento, provendo ações para aumentá-la. Para realizar este acompanhamento, foi criado um indicador de monitoramento da motivação do time: Moving Motivator Cycle Indicator (MCCI).

Abaixo seguem as informações referentes a coleta deste indicador nos três times.

a) Time 1

A figura 1 mostra as pontuações dadas por cada profissional do Time 1 relacionadas a cada motivação ao longo de sua P2Ps:

\begin{tabular}{|c|c|c|c|c|c|c|c|c|c|c|c|c|c|c|}
\hline & \multicolumn{2}{|c|}{ Desenvolvedor $\mathrm{V}$} & \multicolumn{3}{|c|}{ Desenvolvedor $\mathrm{R}$} & \multicolumn{3}{|c|}{ Desenvolvedor $\mathrm{L}$} & \multicolumn{3}{|c|}{ Tester J } & \multicolumn{3}{|c|}{ Tester A } \\
\hline & P2P_1 & P2P_2 & P2P_1 & P2P_2 & P2P_3 & P2P_1 & P2P_2 & P2P_3 & P2P_1 & |P2P_2 & P2P_3 & P2P_1 & P2P_2 & $\mathrm{P} 2 \mathrm{P} 33_{3}$ \\
\hline Curiosidade & 1 & 3 & 2 & & 2 & 1 & 3 & 3 & & 3 & 3 & & & \\
\hline Honra & 3 & 3 & & & 3 & & & & & & 2 & & 2 & 3 \\
\hline Aceitação & & 2 & 3 & & & & & & & & & & 3 & \\
\hline Maestria & 1 & & 2 & & 2 & 2 & & & 1 & 3 & 2 & 2 & & \\
\hline Poder & & & & & & & & & & & & & & \\
\hline Liberdade & & 2 & & & 3 & 3 & 1 & 3 & 3 & 2 & & 3 & 3 & \\
\hline Relacionamento & & & 3 & & & 3 & 3 & 3 & 3 & & & 2 & 3 & 3 \\
\hline Ordem & & & & & & & & & 3 & 2 & 2 & 2 & 3 & 3 \\
\hline Objetivo & 2 & 2 & & & 2 & 1 & 2 & 2 & 3 & 3 & 3 & & & 3 \\
\hline Status & 3 & & 2 & & & & 3 & 1 & & & & 3 & & 2 \\
\hline Somatório Total & 10 & 12 & 12 & & 12 & 10 & 12 & 12 & 13 & 13 & 12 & 12 & 14 & 14 \\
\hline
\end{tabular}

Figura 1. Coleta das P2Ps do Time 1

Observamos que a motivação média estava próxima do máximo e aumentou nas coletas seguintes (ver figura 2(a)), fruto do resultado de ações planejadas individualmente com cada um dos participantes. Na terceira coleta tivemos leve queda, 
motivada pela preocupação de uma profissional (Tester J) com sua maestria, a qual estava sendo desafiada com as novas demandas que surgiram atuando em uma área nova do sistema, em contrapartida sua curiosidade foi elevada com este fato. Observamos isto também com o Desenvolvedor L, que também teve oportunidade de atuar neste novo contexto.

(a)

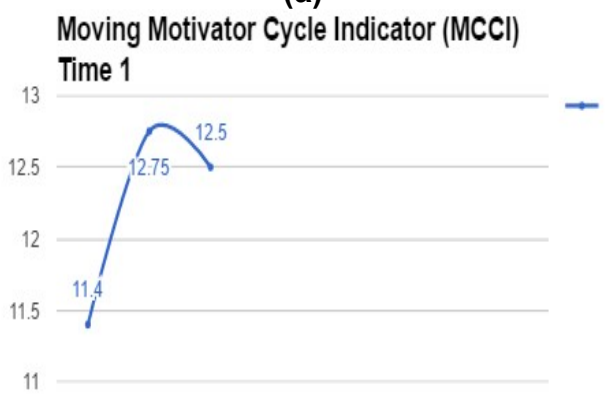

(b)

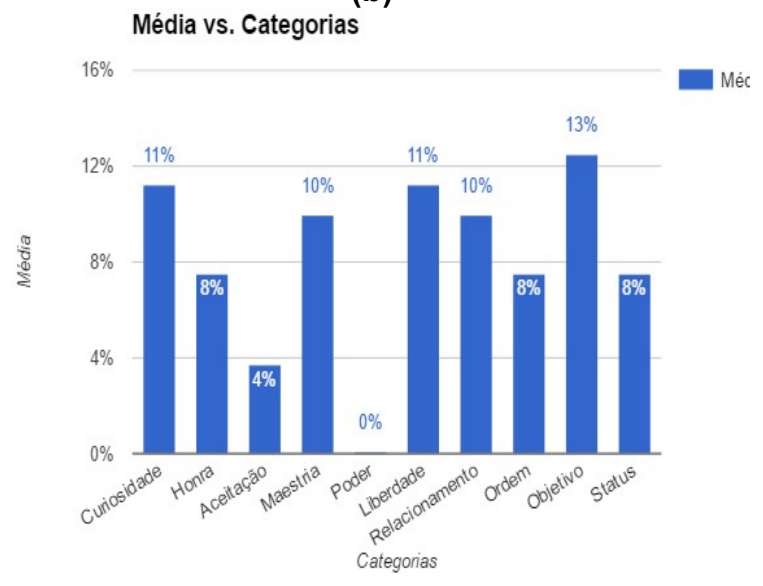

Figura 2. (a) Gráfico Moving Motivator Cycle Indicator (MMCI) do Time 1;

(b) Categorias de motivações mais citadas ao longo das coletas do Time 1.

Outro dado relevante levantado e que pode ser utilizado estrategicamente para promover ações é o uso de uma análise das motivações mais frequentemente citadas, conforme gráfico da Figura 2(b). Vimos que nenhum dos membros almejava poder, o que pode ser motivado por uma característica comum aos membros do time: humildade. $\mathrm{O}$ fato de o motivador mais citado ter sido o objetivo, nos leva a crer que ações para orientar os participantes em suas carreiras surtirá bons efeitos. Outra motivação muito citada foi relacionamento e, como foi observado, houve pontuação muito elevada, fruto de um bom ambiente de trabalho do qual faziam parte.

Como ações, buscamos treinamentos em tecnologia e inglês objetivando atingir a motivação Objetivo e Maestria, as quais foram as principais citadas entre as motivações. Ainda para melhorar Maestria e Objetivo, trabalhamos para conquistar tarefas de maior nível de complexidade e importância no projeto o que elevou também a Curiosidade dos membros do time. Houve participação em provas de conceito.

b) Time 2

As cartas das motivações eram ordenadas em cada P2P e assim eram selecionadas e pontuadas as cinco primeiras. A Figura 3 mostra as pontuações dadas por cada profissional do Time 2 para cada motivação ao longo de suas P2Ps: 


\begin{tabular}{|c|c|c|c|c|c|c|c|c|c|c|c|c|}
\hline \multicolumn{4}{|c|}{ Desenvolvedor A } & \multicolumn{3}{|c|}{ Desenvolvedor B } & \multicolumn{3}{|c|}{ Desenvolvedor C } & \multicolumn{3}{|c|}{ Desenvolvedor D } \\
\hline & $P 2 P_{-} 1$ & $P_{2} P_{-} 2$ & $P_{2} P_{-} 3$ & $P 2 P_{2} 1$ & $P 2 P_{-} 2$ & $P_{2} P_{3} 3$ & $P 2 P_{-} 1$ & $P 2 P_{-} 2$ & $P 2 P_{-} 3$ & $P 2 P_{-} 1$ & $P 2 P_{-} 2$ & $P 2 P_{-} 3$ \\
\hline Curiosidade & 3 & 3 & 3 & & & & 2 & 2 & 2 & & 2 & 3 \\
\hline Honra & 3 & 3 & 3 & 3 & 3 & 3 & 3 & 3 & 3 & & & \\
\hline Aceitação & & & & & & & 3 & 3 & 3 & & 1 & 2 \\
\hline Maestria & 1 & 2 & 2 & 2 & 2 & 2 & & & & & 3 & 3 \\
\hline Poder & & & & 2 & & & & & & & & \\
\hline Liberdade & & & & 3 & 3 & 3 & & & & & & \\
\hline Relacionamento & 3 & 3 & 3 & & & & 3 & 3 & 3 & & & \\
\hline \multicolumn{13}{|l|}{ Ordem } \\
\hline Objetivo & 2 & 2 & 2 & & 2 & 2 & & & & & 1 & 1 \\
\hline \begin{tabular}{|l|} 
Status \\
\end{tabular} & & & & 3 & 3 & 3 & 2 & 2 & 2 & & 1 & 1 \\
\hline Somatório Total & 12 & 13 & 13 & 13 & 13 & 13 & 13 & 13 & 13 & & 8 & 10 \\
\hline
\end{tabular}

Figura 3. Coleta das P2Ps do Time 2

A partir da primeira coleta foi possível acompanhar como esse indicador se comportava a medida que eram executadas as ações traçadas nas P2Ps. Observamos que nas três coletas o indicador ficou acima de $75 \%$ do valor máximo, porém entre a primeira e a segunda coleta o indicador apresentou uma queda (ver figura 2(a)). Isto se deveu ao fato de que a partir da segunda coleta o time recebeu um novo profissional. Este profissional, veio de um outro time onde teve sérios problemas de adaptação e nos primeiros meses da mudança ainda não havia se familiarizado com o novo time. Entre a segunda e a terceira amostra já foi possível observar uma melhora considerável no indicador, fruto das ações realizadas durante este período.

(a)

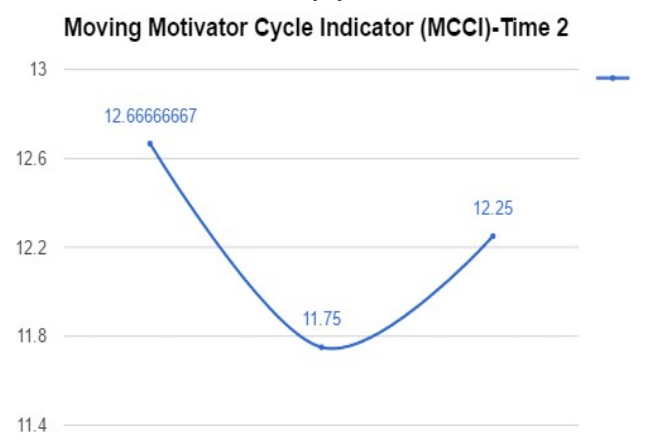

(b)

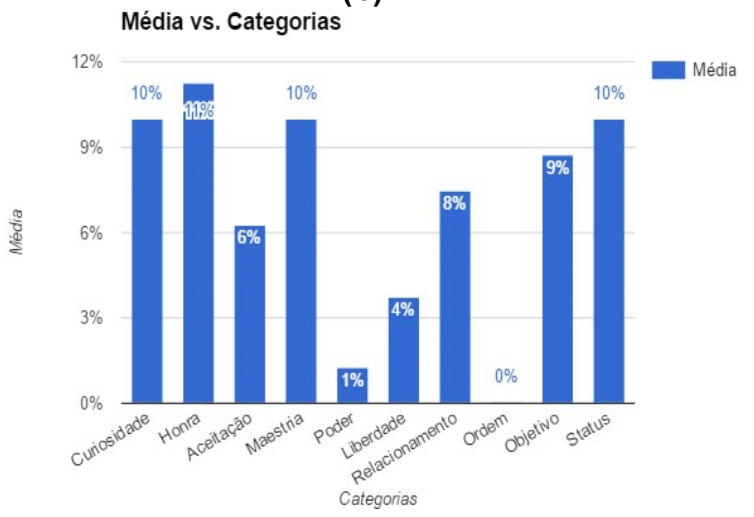

Figura 4. (a) Gráfico Moving Motivator Cycle Indicator ( $\mathrm{MMCl}$ ) do Time 2; (b) Categorias de motivações mais citadas ao longo das coletas do Time 2.

Como esperado, uma vez que o time era misto entre pessoas da geração X e Y, praticamente todas as categorias de motivação foram citadas pelos profissionais do Time 2, conforme Figura 4(b). As categorias menos citadas foram Ordem e Poder, o que pode ser o reflexo de uma característica do time: a simplicidade. $O$ fato de o motivador 
mais citado ser Honra, além de ter recebido a maior pontuação, demonstra que o time tem princípios e que o constante respeito a esses valores deve deixá-los ainda mais satisfeitos e motivados. As outras categorias mais citadas representam um cenário muito comum na área de TI, onde temos profissionais interessados em projetos que os desafie (Curiosidade), instigando a conhecer e se aperfeiçoar mais e mais (Maestria) e consequentemente adquirindo o reconhecimento dos profissionais de seu meio (Status).

Como ações, tivemos treinamentos das tecnologias envolvidas no projeto e atividades para nivelamento do conhecimento nas diversas áreas do projeto colaborando com o aumento da Maestria do time. De uma maneira geral o time tinha interesse em melhorar o aspecto Curiosidade, e sempre que cabível foi dada a oportunidade para de acordo com o seu estilo e preferências realizarem investigações de técnicas e práticas de desenvolvimento para serem analisadas e implementadas no projeto.

c) Time 3

O DEV BS esteve de férias durante a segunda coleta e o DEV R deixou a instituição na terceira coleta. A saída dele está relacionada com o alto nível de importância que este dava ao motivador Objetivo, sendo seu maior objetivo deixar o país. Este profissional também buscava frequentemente Ordem através da implantação de requisitos e com alto nível de detalhe e pequena quantidade de mudanças, o que o projeto não poderia prover pelos fatores de mudança de requisitos frequente $\mathrm{e}$ indefinição nos objetivos. Um fator interessante é que naturalmente este profissional buscava direcionar e influenciar o time, o que apontaria interesse em Poder, mas em nenhuma das duas coletas foi destacado tal motivador. Haviam conflitos de opinião onde o mesmo era o pivô e após sua saída foi possível observar na terceira coleta que 3 dos quatro membros consultados citou que o motivador Relacionamento estava no nível mais alto e apresentava grande importância.

\begin{tabular}{|c|c|c|c|c|c|c|c|c|c|c|c|c|c|c|}
\hline \multicolumn{4}{|c|}{ DEVA } & \multicolumn{3}{|c|}{ DEV BS } & \multicolumn{3}{|c|}{ DEVE } & \multicolumn{2}{|c|}{ DEV R } & \multicolumn{3}{|c|}{ DEV V } \\
\hline & P2P_1 & P2P_2 & P2P_3 & P2P_1 & P2P_ 2 & P2P_3 & Coleta 1 & Coleta 2 & Coleta 3 & P2P_1 & P2P_2 & P2P_1 & P2P_2 & P2P_3 \\
\hline Curiosidade & 2 & 3 & & 3 & & & 3 & 3 & 3 & & & 3 & 3 & 3 \\
\hline Honra & 3 & 3 & 3 & 3 & & 3 & 3 & 2 & 3 & 3 & 3 & 2 & & 2 \\
\hline Aceitação & & & & 2 & & 3 & & & & & & 3 & 3 & 2 \\
\hline Maestria & 2 & 2 & 3 & & & 2 & 3 & 3 & 3 & & & & 2 & 3 \\
\hline Poder & & & & & & & 3 & & & & & & & \\
\hline Liberdade & & & 2 & & & & & 3 & & 3. & 3 & & & \\
\hline Relacionamento & 2 & & 3 & & & 3 & & & 3 & & & 3 & 3. & \\
\hline Ordem & & & 3 & 2 & & & & & & 1 & 1 & & & \\
\hline Objetivo & 2 & 2 & & 1 & & 2 & 2 & 2 & 3 & 3 & 3. & 3 & & 3 \\
\hline Status & & 3 & & & & & & & & 2 & 2 & & 3 & \\
\hline Somatório Total & 11 & 13 & 14 & 11 & & 13 & 14 & 13 & 15 & 12 & 12 & 14 & 14 & 13 \\
\hline
\end{tabular}

Figura 5. Coleta de motivadores do time 3

O DEV A, que inicialmente apresentava o menor nível de motivação, demonstrou grande progresso, posto que este não sentia-se tendo seu potencial explorado (Maestria) por não estar trabalhando em atividades que produziam grande valor agregado. Com o investimento em cursos e o envolvimento do mesmo em atividades de maior nível de importância este elevou ao máximo sua motivação.

Destacamos também que o DEV E apresentou queda na motivação na segunda coleta, pois no auge dos conflitos com o DEV R, a colaboração, seu principal valor (motivador Honra) foi afetado. 
A curva do indicador MMCI foi progressiva, mostrando na terceira coleta um resultado próximo ao valor máximo (quinze), conforme pode ser observado na Figura 6(a).

(a)

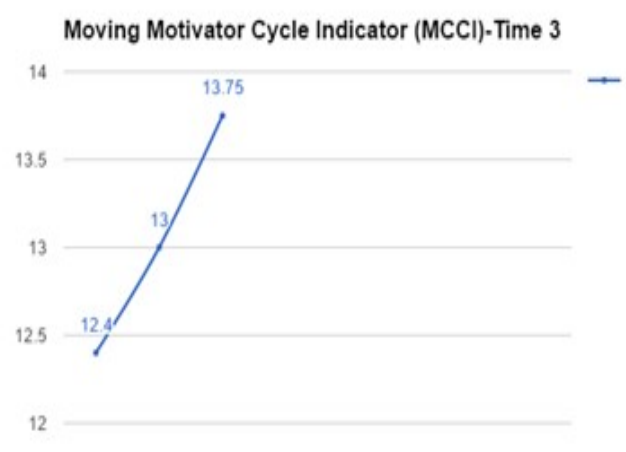

(b)

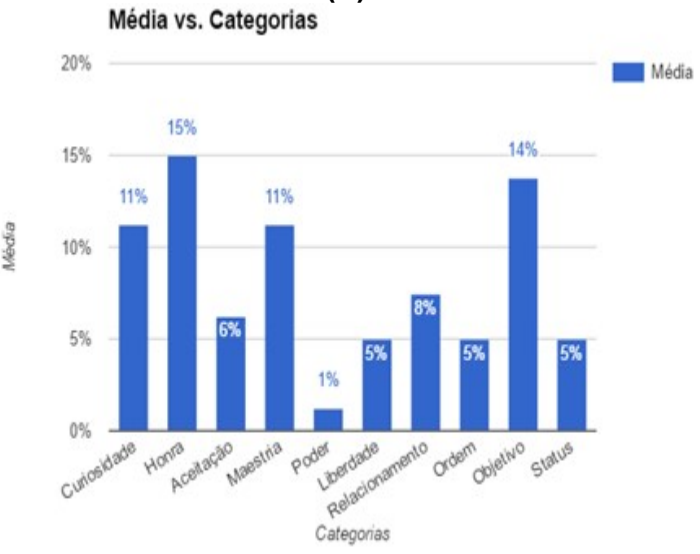

Figura 6. (a) Gráfico Moving Motivator Cycle Indicator ( $\mathrm{MMCl}$ ) do Time 3;

(b) Categorias de motivações mais citadas ao longo das coletas do Time 3.

Os dois motivadores mais citados, Honra e Objetivo, ver figura 6(b), sofreram progresso ao longo das coletas, o que foi o motivador da subida do indicador MMCI. Houve nesse sentido melhora na colaboração entre os membros do time e investimentos para melhorar os conhecimentos dos membros do time no sentido de trazer progressões em suas carreiras, o que trouxe progressos também para a terceira motivação mais citada: Maestria.

\section{Conclusão e Trabalhos Futuros}

A ferramenta proposta neste artigo traz uma análise mais assertiva sobre o monitoramento da motivação de pessoas, um desafio que é recorrente aos gestores e líderes. O diálogo e a compreensão foram facilitados entre as pessoas.

Foi possível observar que tivemos uma maior facilidade de entender as necessidades dos membros do time, uma vez que o uso da ferramenta guia nossa conversa e levanta pontos que talvez não tenham sido observados antes. A instituição também passou a sensibilizar os indivíduos quanto à preocupação recorrente com os mesmos, fazendo aumentar a gratidão dos profissionais, o que foi constatado através de feedbacks dos participantes. A ferramenta atua também como uma forma do próprio profissional analisar de forma mais consistente suas prioridades.

A ferramenta trouxe aos profissionais também um maior reconhecimento das motivações apresentadas no ambiente de trabalho, os quais muitas vezes passam despercebidos no dia-a-dia, o que naturalmente o fará refletir sobre as vantagens de atuar em tal ambiente. 
Com o Moving Motivator Cycle Indicator, é possível tornar palpável o monitoramento da motivação intrínseca do time, fazendo um acompanhamento mais estratégico da sua motivação média. A alta gestão da instituição passou a contar com um dado concreto quanto à motivação de funcionários através deste indicador.

Embora não tenha sido aplicado durante este estudo de caso, o acompanhamento das motivações mais citadas entre os times pode fazer com que as empresas possam traçar ações gerais que afetem grandes quantidades de pessoas.

Uma vez que a grande maioria dos participantes pertence a geração $\mathrm{Y}$, foi possível verificar o grande nível de importância que estes dão às motivações intrínsecas, posto que todos, quando perguntados, responderam que o acompanhamento destas apresentava um grande nível de relevância.

Como proposta de trabalhos futuros podemos citar:

- Utilização da técnica MMC como insumo para a análise de performance dos projetos, verificando de fato o reflexo das ações e melhorias levantadas com os indivíduos nos indicadores de desempenho dos projetos;

- Análise das motivações intrínsecas em relação às motivações extrínsecas;

- Ponderação dos resultados de acordo com a ordem de importância escolhida pelo profissional para as motivações;

- Estabelecer uma meta para o indicador MMCI, verificando o histórico de coletas dos profissionais;

- Realizar a análise das motivações mais citadas entre todas as equipes objetivando propor ações para afetar vários funcionários.

\section{Referências}

Appelo, J. (2011). Management 3.0: leading Agile developers, developing Agile leaders. Pearson Education.

Beck, Kent, et al. (2001) "Manifesto for agile software development.".

BOX1824 (2013). Millennials or Generation Y, Who They Are and Why The're Hated. Disponível em: $<$ https://www.youtube.com/watch? $=\mathrm{v} H$ HpbdQCMnwQ $>$. Acesso em 18 fev. 2016.

Castka, P., Bamber, C.J., Sharp, J.M. e Belohoubek, P. (2001) "Factors affecting successful implementation of high performance teams", Em: Team Performance Management: An International Journal, volume. 7, n: 7/8, p.123 - 134.

Chiavenato, I. (2008). Gestão de pessoas. Elsevier Brasil.

Cox, W. M., e Klinger, E. (Eds.) (2004). Handbook of motivational counseling: Concepts, approaches, and assessment. John Wiley \& Sons.Pires, C. G., Marinho F. G., Souza, G.T. e Belchior, A. D. (2004). "A Experiência de Melhoria do Processo do Instituto Atlântico Baseado no SW-CMM nível 2”, III SBQS, Brasília. 
De Oliveira, G. F. (2015). LIDERANÇA DA GERAÇÃO Y: OS DESAFIOS DO MERCADO DE TRABALHO. Novatio Iuris, 4(2).

Ferreira, Camila L. e Ramos, Bruno S. (2010) "O aumento da produtividade através da valorização dos colaboradores: uma estratégia para a conquista de mercado." Em: Revista de Engenharia e Tecnologia, volume 2, n. 2, p. 71-80.

Jiang, B., Baker, R. C., e Frazier, G. V. (2009). An analysis of job dissatisfaction and turnover to reduce global supply chain risk: Evidence from China. Journal of Operations Management, 27(2), p. 169-184.

Knapik, Janete (2011). Gestão de Pessoas e Talentos, Ibpex, p. 17-19.

Lindström, Kari (1994). "Psychosocial criteria for good work organization", Em: Scandinavian Journal of Work, Environment \& Health, Twentieth Anniversary Issue, Volume 20, p. 123-133.

Lipkin, N. A. e Perrymore, A. (2010). A geração y no trabalho: como lidar com a força de trabalho que influenciará definitivamente a cultura da sua empresa. Elsevier.

Periard, Gustavo (2011). "Tudo sobre a Teoria dos Dois Fatores de Frederick Herzberg". Disponível em $<$ http://www.sobreadministracao.com/tudo-sobre-a-teoriados-dois-fatores-de-frederick-herzberg/>. Acesso em 18 fev. 2016.

Pink, D. H. (2011). Drive: The surprising truth about what motivates us. Penguin.

Pires, C. G., Marinho, F. G., Souza, G.T. e Belchior, A. D. (2004), "A Experiência de Melhoria do Processo do Instituto Atlântico Baseado no SW-CMM nível 2", III SBQS, Brasília.

PMI - Project Management Institute. (2012), Um guia do conjunto de conhecimentos do gerenciamento de projetos (guia PMBOK). $5^{\mathrm{a}}$ ed. Project Management Institute, Pennsylvania.

Ramlall, S. (2004). A review of employee motivation theories and their implications for employee retention within organizations. Em: Journal of American Academy of Business, volume 5, n. 1/2, p. 52-63.

Reiss, Steven (2002), "Who Am I? The 16 Basic Desires That Motivate Our Actions and Define Our Personalities", Berkley Trade.

Ryan, Richard M. e Deci, Edward L. (2000). "Intrinsic and Extrinsic Motivations: Classic Definitions and New Directions", Em: Contemporary Educational Psychology, Elsevier, Volume 25, $1^{\text {a }}$ edição, p. 54 - 67.

Stott, K., \& Walker, A. (1995). Teams, Teamwork and Teambuilding: The Manager's Complete Guide to Teams in Organisations. Prentice Hall.

Thatcher, J. B., Liu, Y., Stepina, L. P., Goodman, J. M. e Treadway, D. C. (2006). IT worker turnover: An empirical examination of intrinsic motivation. ACM SIGMIS Database, 37(2-3), p. 133-146. 